\title{
Effect of the Water Soluble Fraction on the Viscoelasticity of Wheat Gluten
}

\author{
Chiaki YOSHIDA and Gen-ichi DANNO* \\ Kyoto Junior College, Hori, Fukuchiyama 620, Kyoto, Japan \\ * Department of Agricultural Chemistry, Kobe University, \\ Nada-ku, Kobe 657, Japan
}

Received July 18, 1988

\begin{abstract}
The effects of the water soluble fraction and the dough mixing procedure on the formation of a viscoelasic gluten mass were examined by means of a reconstitution study. Gluten and the water soluble fraction were prepared by two methods, water extraction and dough mixing, and then lyophilized. With the dough mixing method, water soluble components, especially gliadin, were incorporated into the gluten mass. On the other hand, gluten prepared by water extraction was poor in gliadin and so, being elastic and less extensible, differing from gluten obtained from dough.

Gluten was prepared from reconstituted dough, in which lyophilized gluten, lyophilized water soluble fraction and starch were mixed in the original ratio. The reconstituted gluten with gluten obtained by dough mixing had the same rheological properties as those of gluten from flour. The same results were obtained when gliadin was used instead of the water soluble fraction. On the other hand, despite the same ratio of gliadin and glutenin, the reconstituted gluten with gluten obtained by water extraction was elastic and less extensible. These lines of evidence suggested that for the formation of a viscoelastic mass, gliadin must be well mixed with glutenin to form a homogeneous gluten network.

In this paper, we also found that gliadin was one of the factors causing breakdown and this phenomenon was related to the formation of a viscoelastic mass during dough mixing.
\end{abstract}

Dough is composed of gluten, starch, lipids and low molecular weight substances. Its rheological properties are affected by many factors, the mixing conditions, and the quality and quantity of gluten proteins. The water soluble fraction is also a factor affecting dough properties. It has been shown in reconstitution studies that dough without the water soluble fraction has different mixing characteristics from the original dough. ${ }^{1 \sim 3)}$ No breakdown occurred in the reconstituted dough without the water soluble fraction, but similar properties were recovered on the addition of the water soluble fraction, such as a reduction in the mixing time and in the stability of the dough. As the mixing characteristics may be dependent on the formation of a viscoelastic gluten mass during dough mixing, it is plausible that the water soluble fraction affects the viscoelasticity of gluten in dough.

In a preliminary experiment, we noticed that when flour was extracted with sufficient water, the gluten obtained was dispersible and less aggregative. The gluten mass collected on centrifugation was elastic, differing from the gluten mass obtained from dough. There are two possible reasons for the failure in the formation of a viscoelastic mass; the removal of the water soluble fraction and the procedure without dough mixing.

In the present experiment, we tried to clarify how these factors are concerned with the formation of a viscoelastic gluten mass by means of a reconstitution study.

\section{Materials and Methods}

Flour. Commercially milled flour made from Canada Western. wheat No. 1 (1CW) was donated by Nisshin Milling Co. The flour was defatted with $n$-butanol and then air-dried.

Dough. For a small scale experiment, dough was pre- 
pared in a mortar with gentle hand mixing. The mixing profile was similar to that in the case of mechanical mixing $(60 \mathrm{rpm})$ with a Bench Kneader, although a high shear force as in the case of mechanical mixing was avoided. The dough became soft after $3 \mathrm{~min}$ mixing and the dough after $8 \mathrm{~min}$ mixing was used in this experiment, which will correspond to overmixed dough.

Protein Fractionation. Two methods, Methods I and II, were employed for the preparation of gluten as follows. For Method I, $10 \mathrm{~g}$ of flour was suspended in $150 \mathrm{ml}$ water and then stirred for $30 \mathrm{~min}$ at room temperature. The slurry was centrifuged at $3000 \mathrm{rpm}$ for $5 \mathrm{~min}$ to remove the water soluble fraction. This procedure was repeated once more and then the residue was separated into the gluten mass and starch. The gluten mass was lyophilized and designated as G-I. The combined water soluble fraction was also lyophilized and designated as WS-I. The yields of G-I and WS-I were 0.5 and $0.4 \mathrm{~g}$, respectively. For Method II, dough from $10 \mathrm{~g}$ flour was extracted five times with a 6 fold amount of water. The gluten mass formed was lyophilized and designated as G-II. The extract was centrifuged at $8000 \mathrm{rpm}$ for $20 \mathrm{~min}$ to remove residual starch. The water soluble fraction obtained was lyophilized and designated as WS-II. The yields of G-II and WS-II were 0.65 and $0.25 \mathrm{~g}$, respectively. The protein contents of $\mathrm{G}-\mathrm{I}$ and G-II were 70.6 and $69.8 \%$, respectively, so starch included in the two lyophilized glutens was nearly the same in amount. Gliadin and glutenin were fractionated from wet gluten according to the method of Jones et al. ${ }^{4)}$ The acetic acid insoluble fraction was solubilized in $2 \% 2$ mercaptoethanol for $24 \mathrm{hr}$ at $5^{\circ} \mathrm{C}$. Protein was determined by the micro-Kjeldahl method $(\mathrm{N} \times 5.7)$.

$S D S-P A G E$. SDS-polyacrylamide gel electrophoresis (SDS-PAGE) was performed according to the method of Weber et al. ${ }^{5)}$

Rheological Test. Rheological properties were measured with a Tensipresser (Takemoto Inc.). For consistency measurement, $7.5 \mathrm{~g}$ of dough was molded into a disk of
$1.5 \mathrm{~cm}$ thickness (diameter, $2.5 \mathrm{~cm}$ ). The measurement was performed with a plunger of $18 \mathrm{~mm}$ diameter, with $7 \mathrm{~mm}$ clearance. In the case of gluten, $1.5 \mathrm{~g}$ of gluten was molded into a disk of $1 \mathrm{~cm}$ thickness (diameter, $1.3 \mathrm{~cm}$ ), with $2 \mathrm{~mm}$ clearance. For extensibility measurement, $1.5 \mathrm{~g}$ of gluten was molded into a rectangle of $3 \mathrm{~mm}$ thickness (cross section, $0.3 \mathrm{~cm}^{2}$ ) and both edges were fixed with clamps. The extension test was performed with a hook of $5 \mathrm{~mm}$ diameter in a similar manner to in the case of an Extensigraph. Reconstituted dough was prepared so as to have a protein content equal to that of the original flour, with the addition of lyophilized gluten, lyophilized water soluble fraction and starch.

\section{Results}

Gluten prepared by water extraction was too dispersible to form a gluten mass, so it was collected by centrifugation. The gluten mass obtained was elastic and poor in extensibility, differing from the gluten mass obtained from dough. Typical photographs are shown in Fig. 1. The gluten obtained by water extraction dispersed into small particles and had almost no extensibility, as shown in Fig. 2. The rheological properties of the collected gluten mass were characterized by a high consistency and less extensibility. Further kneading of the mass resulted in enhanced elasticity (data not shown). However, when immersed in water and gently kneaded by hand, the gluten mass again became dispersible, so the change in the rheological properties was reversible. Therefore, the enhanced elasticity should be due to the decrease in water content, and not to any chemical reactions such as the formation of disulfide bonds.

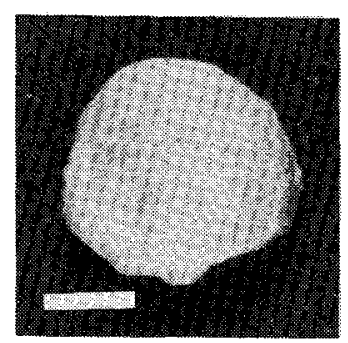

a

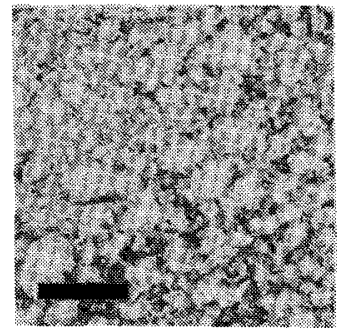

b

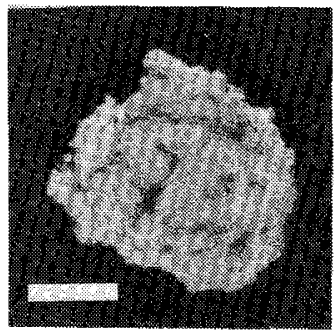

C

Fig. 1. Photographs of Glutens.

$a$, gluten from dough; b, gluten obtained on water extraction; $c$, gluten after centrifugation of $b$. Bar $=1 \mathrm{~cm}$. 


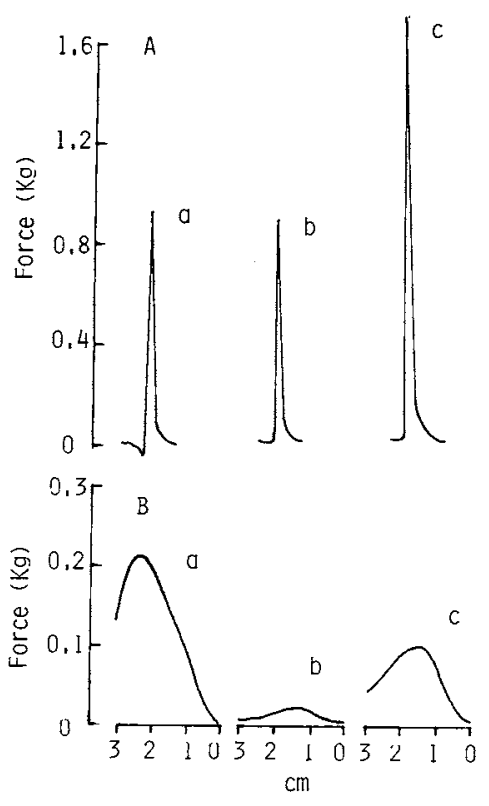

Fig. 2. Rheological Properties of Glutens.

A. Consistency of gluten. The procedures were as described under Materials and Methods. The ordinate represents the force exerted by the plunger.

$a$, gluten from dough; $b$, gluten obtained on water extraction; c, gluten after centrifugation of $b$.

B. Extensibility of gluten. The procedures were as described under Materials and Methods. The ordinate and abscissa represent the force exerted by the hook and the distance moved by the hook, respectively.

$a$, gluten from dough; b, gluten obtained on water extraction; $c$, gluten after centrifugation of $b$.

Table I. Comparison of the Protein Compositions WITH METHODS I AND II

\begin{tabular}{lcc}
\hline & \multicolumn{2}{c}{${\text { Protein }(\mathrm{mg})^{a}}^{a}$} \\
\cline { 2 - 3 } & Method I & Method II \\
\hline Water soluble & 224 & 120 \\
Gluten & 776 & 1040 \\
Gliadin & 393 & 581 \\
Glutenin & 263 & 374 \\
Insoluble & 108 & 12 \\
\hline
\end{tabular}

a From $10 \mathrm{~g}$ wheat flour.

It is expected that the enhanced elasticity will be related to the decrease in water retention of the gluten mass, concomitant with the removal of the water soluble fraction. To confirm this, the composition of protein ob-

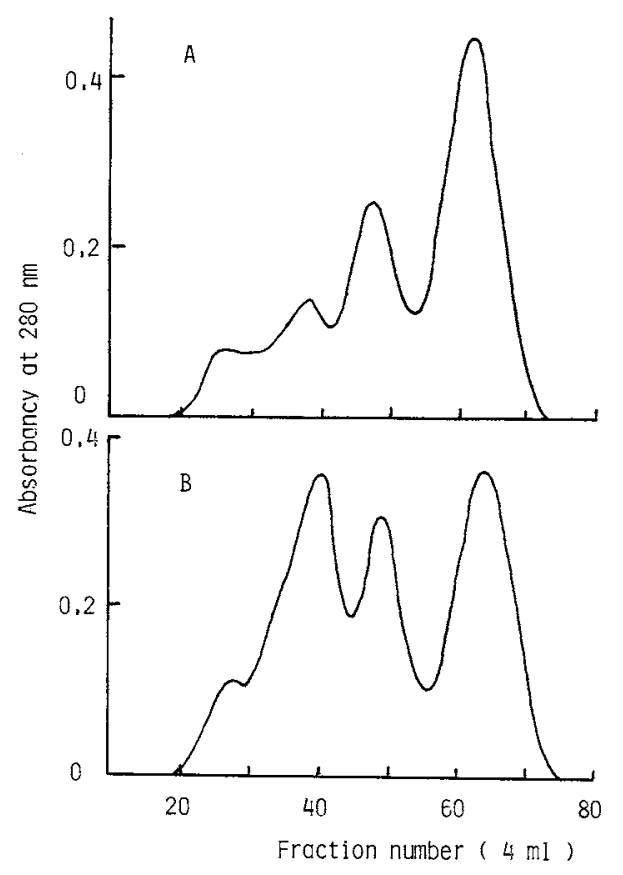

Fig. 3. Gel Filtration Patterns of Water Soluble Fractions Eluted from a Sephadex G-100 column $(2.6 \times 40 \mathrm{~cm})$.

The column was equilibrated with $0.1 \mathrm{~N}$ acetic acid and the flow rate was $20 \mathrm{ml} / \mathrm{hr}$ at room temperature. One hundred $\mathrm{mg}$ of the water soluble fraction was dissolved in $4 \mathrm{ml}$ of $0.1 \mathrm{~N}$ acetic acid and applied to the column.

A, water soluble fraction from dough; B, water soluble fraction obtained on water extraction.

tained from mixed dough (Method II) was compared with that prepared by water extraction (Method I). As shown in Table I, a decrease in the water soluble fraction and an increase in gluten accompanied the dough mixing. The ratio of gliadin to glutenin was slightly higher than that in the case of water extraction. Another feature was the disappearance of acid insoluble protein with the dought mixing method. This phenomenon is consistent with many reports and is related to the increase in extractability during dough mixing. $^{6 \sim 8}$ )

\section{Gel filtration and SDS-PAGE pattern}

The protein constituents in the water soluble fraction and gluten were determined by gel filtration on a Sephadex G-100 column 

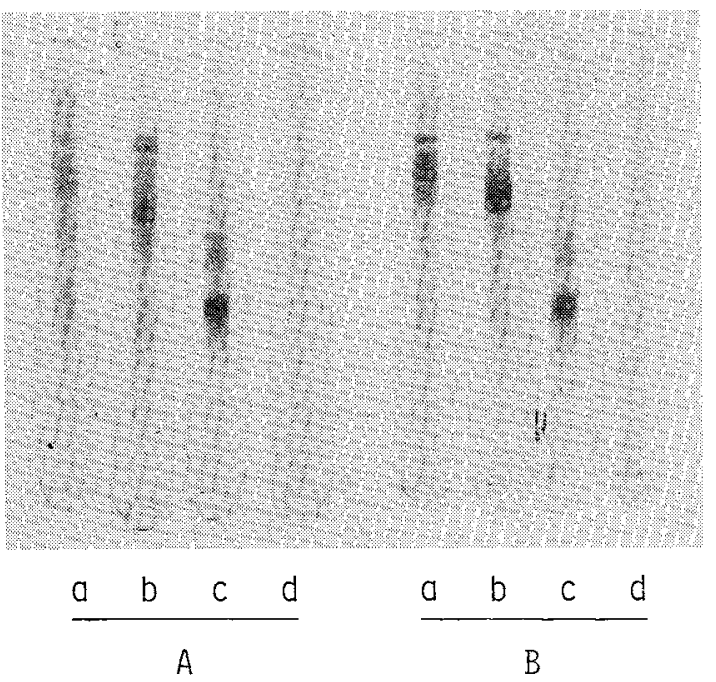

Fig. 4. SDS-PAGE Patterns of Eluates on Gel Filtration of Water Soluble Fractions.

The procedures were as described under Materials and Methods.

A. Eluates of the water soluble fraction from dough: a, first peak; $b$, second peak; $c$, third peak; $d$, fourth peak. B. Eluates of the water soluble fraction obtained on water extraction: a, first peak; b, second peak; $c$, third peak; $d$, fourth peak.

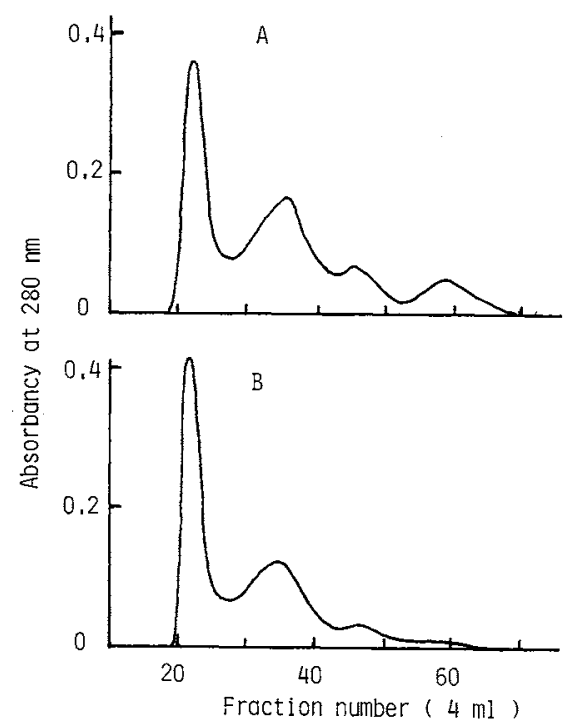

Fig. 5. Gel Filtration Patterns of Glutens Eluted on a Sephadex G-100 column $(2.6 \times 40 \mathrm{~cm})$.

Four $\mathrm{ml}$ of protein solution (total protein, $20 \mathrm{mg}$ ) extracted from wet gluten was applied to the column.

A, gluten from dough; B, gluten obtained on water extraction.

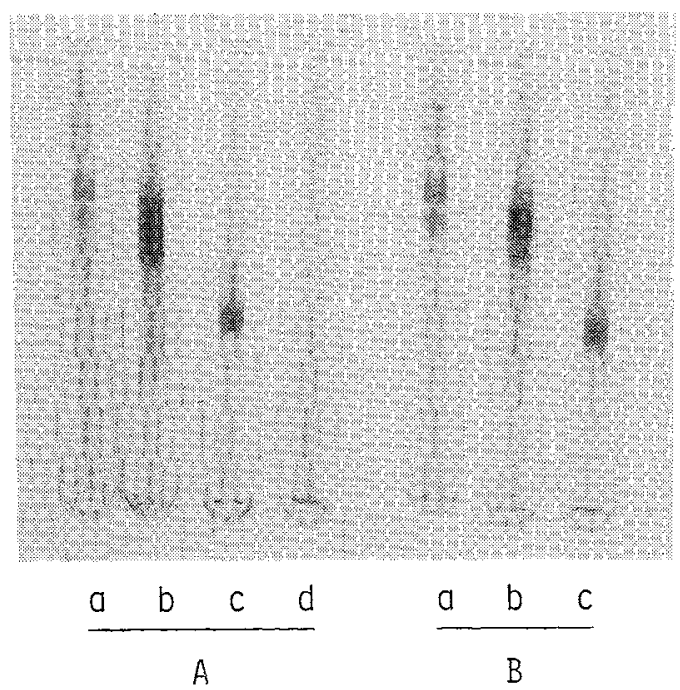

Fig. 6. SDS-PAGE Patterns of Eluates on Gel Filtration of Glutens.

The Procedures were as described under Materials and Methods.

A. Eluates of gluten from dough: a, first peak; $b$, second peak; c, third peak; d, fourth peak.

B. Eluates of gluten obtained on water extraction: a, first peak; $b$, second peak; $c$, third peak.

$(2.6 \times 40 \mathrm{~cm})$ and SDS-PAGE. As shown in Fig. 3 , the gel filtration patterns of both water soluble fractions showed the presence of four components. A striking difference was seen in the decrease in the second component in the water soluble fraction from dough. The SDSPAGE pattern showed that this component was comprised of gliadin, as shown in Fig. 4, as reported by Mullen and Smith. ${ }^{9)}$ The first, third and fourth peaks were contaminating gliadin, albumin plus globulin and non-protein components, respectively. The elution pattern of gluten and the SDS-PAGE pattern of gluten are shown in Figs. 5 and 6 , respectively. The elution pattern was quite similar to that reported by Meredith and Wren. ${ }^{10)}$ Each component corresponded to that on gel filtration of the water soluble fraction. The elution patterns of the two glutens were similar except for the higher amounts of the third and fourth components in gluten from dough. These lines of evidence indicate that water soluble components, particularly gliadin, were incorpo- 


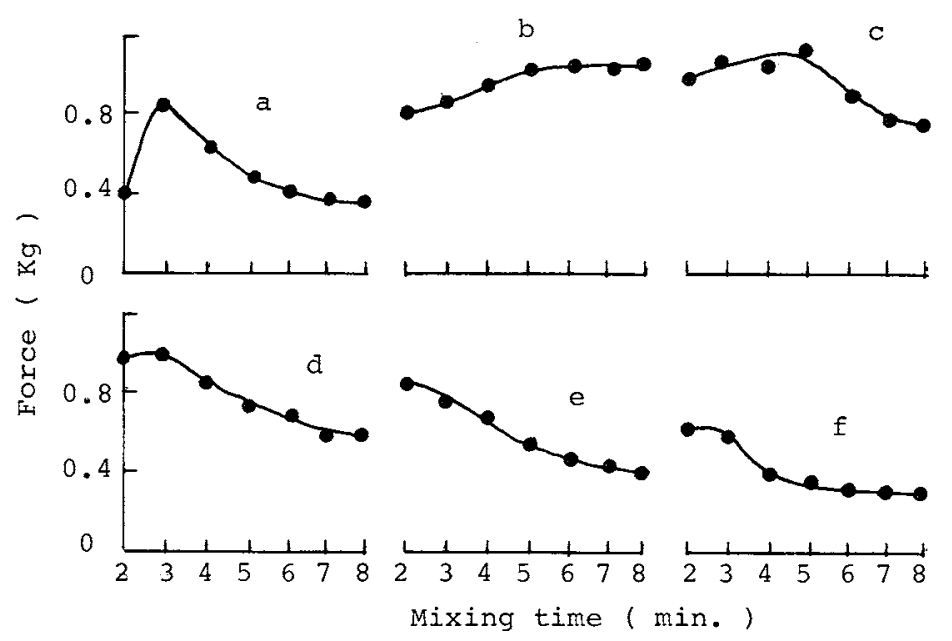

Fig. 7. Texture Profiles of Dough and Reconstituted Doughs.

The procedures were as described under Materials and Methods.

a, dough; b, reconstituted dough with G-I; c, reconstituted dough with G-I plus WS-I; d, reconstituted dough with G-II; e, reconstituted dough with G-II plus WS-II; f, reconstituted dough with G-II plus gliadin.

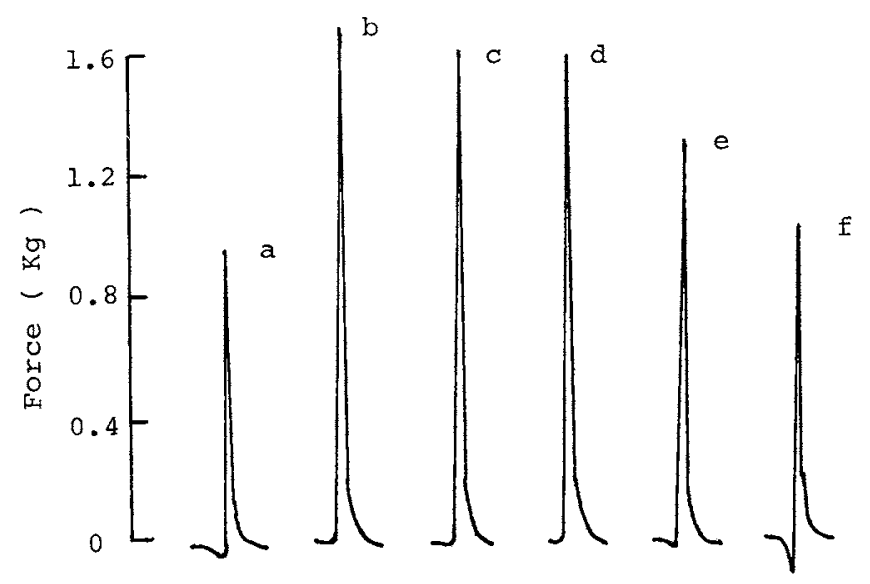

Fig. 8. Consistency of Gluten and Glutens from Reconstituted Doughs.

The procedures were as described under Materials and Methods.

a, gluten from dough; $b$, gluten from reconstituted dough with G-I; c, gluten from reconstituted dough with GI plus WS-I; d, gluten from reconstituted dough with G-II; e, gluten from reconstituted dough with G-II plus WS-II; f, gluten from reconstituted dough with G-II plus gliadin.

rated into the gluten mass during dough mixing. In other words, the gluten mass on water extraction was poor in gliadin, resulting in the formation of an elastic mass.

\section{Rheological properties}

The effect of the water soluble fraction on the formation of a viscoelastic mass was exam- ined by means of a reconstitution study. Figure 7a shows the texture profile or of dough during mixing. To $5 \mathrm{~g}$ of flour was added $2.5 \mathrm{ml}$ of water, followed by mixing by hand for two minutes, and then the consistency was measured immediately. Subsequent measurements wre performed at one minute intervals during discontinuous mixing. Despite the different 
mixing procedure, the profile was similar to a Mixogram reported elsewhere ${ }^{3)}$; the consistency became maximum at about $3 \mathrm{~min}$, followed by a gradual decrease. So, the hand kneading employed in this experiment should be as adequate as the prevailing method. The gradual decrease in consistency will correspond to the breakdown seen in a Mixogram. In reconstituted dough with G-I, no breakdown occurred without the addition of WS-I, as noted by Schroeder and Hoseney. ${ }^{31}$ In contrast, when G-II was used, breakdown was observed in spite of the absence of WS-II. This will be due to the fact that the water soluble fraction was already incorporated into G-II. With the addition of WS-II or gliadin, a similar profile to that in the case of dough from flour was obtained. This caused the

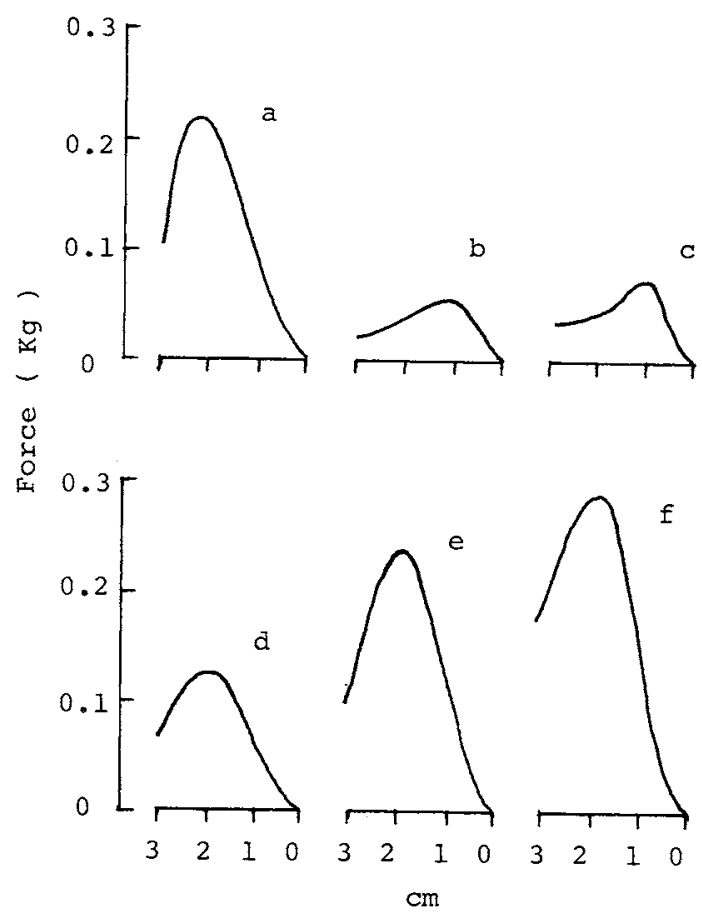

Fig. 9. Extensibility of Gluten and Glutens from Reconstituted Doughs.

The procedures were as described under Materials and Methods.

a, gluten from dough; $b$, gluten from reconstituted dough with G-I; c, gluten from reconstituted dough with G-I plus WS-I; d, gluten from reconstituted dough with G-II; e, gluten from reconstituted dough with G-II plus WS-II; $f$, gluten from reconstituted dough with G-II plus gliadin. decrease in the consistency in comparison to the original dough level. Therefore, it was suggested that gliadin is one of the factors causing breakdown. Figure 8 shows the consistency of gluten from reconstituted dough. As observed for the gluten obtained on water extraction, the glutens from reconstituted doughs with G-I alone, and G-I plus WS-I were both dispersible and hard to aggregate, and so they were collected by centrifugation after washing out most of the starch. On the other hand, gluten from reconstituted dough with G-II was easily collected and formed a viscoelastic mass. The consistency of gluten with G-I alone was higher than that of gluten from flour. The addition of WS-I to the reconstituted dough was not effective, despite there being the same protein constituents as in flour. In the case of G-II, comparable consistency with that of gluten from flour was obtained with the addition of WS-II or gliadin. Without the addition of WS-II, the consistency was higher, probably due to the release of the water soluble fraction from G-II during dough mixing. So, excess water soluble fraction will be needed for the formation of a viscoelastic mass. Figure 9 shows the extensibility of gluten from reconstituted dough. The gluten with G-I alone was less resistant as to tensile force, easily broken and poor in extensibility. The fragility was not improved by the addition of WS-I. On the other hand, in the case of G-II, almost the same extensibiliy was recovered on the addition of WS-II or gliadin. The absence of WS-II resulted in lower extensibility.

\section{Discussion}

In this study, we examined the effects of the water soluble fraction and the mixing procedure on the formation of a viscoelastic mass. Glutenin is known to be an elastic mass, whereas gliadin is known to be a viscous mass. So, the ratio will affect the viscoelasticity of the gluten mass. About $30 \%$ of gliadin was solubilized in water when flour was extracted with sufficient water (Table I). However, a considerable amount was found in the gluten mass, 
probably due to the limited solubility and high affinity to glutenin (Fig. 3). Under dough mixing conditions, additional gliadin was incorporated into the gluten mass. G-I rich in glutenin was more elastic than G-II rich in gliadin, as expected. So, an appropriate amount of gliadin in the gluten mass will be responsible for the viscoelasticity. The effect of the water soluble fraction of dough mixing characteristics has been clarified in reconstitution studies ${ }^{1 \sim 3}$; the water soluble fraction shortened the mixing time and caused breakdown. The same results were obtained in our study with reconstituted dough, supporting the adequacy of our experimental method as to dough mixing (Fig. 7).

The another factor, the dough mixing procedure, was found to be required for the formation of a viscoelastic mass in the reconstitution study. The reconstituted dough with both G-I plus WS-I and G-II plus WS-II had a protein content equal to that of the original flour. Despite the same ratio of glutenin and gliadin, the dough with G-I plus WS-I did not form a viscoelastic mass (Figs. 8 and 9). The failure in mass formation should be due to nomixing in preparation of the gluten mass. In this connection, it was observed that when reconstituted gluten was prepared in the $a b$ sence of starch, the gluten mass in the case of G-I plus WS-I had the same rheological properties as that from flour, and with G-II plus WS-II (data not shown). However, it required a longer mixing time for the formation of a viscoelastic mass. In G-I, glutenin and gliadin will not be well mixed, so longer mixing is required for the formation of a homogeneous mixture of glutenin and gliadin. In this case, the presence of starch prevented the formation, probably disturbing the rearrangement of glutenin and gliadin during dough mixing. Instead of the water soluble fraction, the addition of the third (albumin and globulin) and fourth (non-protein fraction) components obtained on gel filtration did not allow reproduction of the viscoelastic mass (data not shown). So, water soluble components other than gliadin may not be effec- tive. On the other hand, a viscoelastic mass was obtained with reconstituted dough with G-II plus WS-II, or G-II plus gliadin. In this case, starch did not prevent the formation. In G-II, glutenin and gliadin will be well mixed to form a mosaic structure composed of two proteins, as proposed by Wall ${ }^{11)}$ and Huebner. ${ }^{12)}$ Gliadin must be well mixed with glutenin to form a homogeneous gluten network for the formation of a viscoelastic mass. Removal of the effective gliadin by water extraction produces an elastic mass rich in glutenin. The acid insoluble glutenin formed in the case of water extraction may result from strong glutenin-glutenin interactions.

Many explanations have been given for the breakdown. In this experiment, we propose that the breakdown may be related to the formation of a viscoelastic mass during mixing. The gradual decrease in consistency during mixing corresponds to the breakdown seen in a Mixogram. Breadkown was observed in reconstituted dough, from which the viscoelastic gluten mass was produced. On the other hand, no breakdown was observed in reconstituted dough with G-I. The gluten obtained was not viscoelastic, but dispersible. Although the addition of WS-I caused breadkdown slightly, the mixing was not enough for the formation of a viscoelastic mass. Further mixing will give the same result as in the case of the original dough. Starch will prevent the formation of a gluten network. These lines of evidence suggest that breakdown will be caused by the formation of a viscoelastic mass during dough mixing.

\section{References}

1) D. E. Smith and J. D. Mullen, Cereal Chem., 42, 275 (1965).

2) P. J. Mattern and R. M. Sandstedt, Cereal Chem., 39, 81 (1962).

3) L. F. Schroeder and R. C. Hoseney, Cereal Chem., 55, $348(1978)$

4) R. W. Jones, N. W. Taylor and F. R. Senti, Arch. Biochem. Biophys., 84, 363 (1959).

5) K. Weber, J. R. Pringle and M. Osborn, in "Methods in Enzymology," Vol. 26, ed. by S. P. Colowick and N. O. Kaplan, Academic Press, New York, 1972, p. 3. 
6) D. K. Mecham, Baker's Dig., 38(2), 46 (1964).

7) C. C. Tsen, Cereal Chem., 44, 308 (1967)

8) G. Danno and R. C. Hoseney, Cereal Chem., 59, 196 (1982).

9) J. D. Mullen and D. E. Smith, Cereal Chem., 42, 263 (1965).
10) O. B. Meredith and J. J. Wren, Cereal Chem., 48, 169 (1966).

11) J. S. Wall, in "Recent Advances in the Biochemistry of Cereals," ed. by D. L. Laidman and R. G. Wyn Jones, Academic Press, New York, 1979, p. 275.

12) F. R. Huebner, Baker's Dig., 51(5), 25 (1977). 